\title{
SOFT MODELING OF KNOWLEDGE SYSTEMS THROUGH FUZZY PETRI NETS
}

Felipe Lara-Rosano

Centre for Applied Sciences and Technological Development, National University of Mexico

e-mail: lararf@servidor.unam.mx

Abstract: Knowledge in the social and human Sciences cannot be modeled with old time mathematical modeling techniques. In this situation, it would be very useful to have modeling approaches capable to express the richness of social reality, including its vagueness and uncertainty. Aiming at this objective, a soft modeling approach is proposed, implemented as a generalized fuzzy Petri net. This model can be then used to make inferences and to solve problems related to the complex system.

Key words: Complex systems, soft models, fuzzy Petri nets, knowledge-based systems, knowledge representation.

\section{INTRODUCTION}

Real problems in the social and human environments in a modern world are characterized by a great economic polarisation of societies, aggresive global markets, environmental problems, natural resources depletion, violence and crime outbursts, etc. These complex problems cannot be approached with old time mathematical modeling techniques. In the 50's and 60's the Expert Consultation Techniques were introduced as valid heuristic methods to analyze and to handle social problems [1]. These techniques are based not on trying to model mathematically the complex real world, but to extract the conceptual logical model that an expert or a panel of experts have 
about it, that permits them with remarkable efficiency to solve real world problems in their speciality domains. This conceptual logical model is expressed in terms of rules of behavior of the system elements, as a Knowledge Base, that is a collection of logical propositions whose relationships model the knowledge that experts have about a certain topic.

One of the principal issues in building Knowledge Bases is related to modeling real situations characterized by uncertainty [2]. This uncertainty may be produced by following factors: [3]:

a) It is impossible to assign the whole truth or the whole falsity to propositions, even to those taken as premises or starting points of a logical discourse [4].

b) The logical support of a premise or condition for determining a given conclusion or result is uncertain, that is, we can define a conditional belief value of a conclusion given a premise.

c) The premises contain fuzzy terms.

A way to model this fuzzy logic model is the fuzzy production rule. The components of a fuzzy production rule are:

a) A conclusion whose belief value is to be estimated.

b) The premises and their belief values.

c) The logical combination of the premises, generally expressed in a normal form as a disjunction of conjunctions.

d) The uncertain implication relating the belief value of the conclusion with the premises.

The logical belief functions should be drawn from a specific fuzzy logic. In a former paper Lara-Rosano [3] proposed the Logic of Lukasiewicz as the most suitable one to handle uncertainty according to a set of logic-mathematical criteria.

In this paper a Fuzzy Petri Net approach is proposed to model linguistic conceptual models, using linguistic belief values for the propositions, as well as a linguistic weight for the contributions of premises to the belief value of the conclusion. This kind of model can be used to make inferences and to solve problems related to the complex system. 


\section{LINGUISTIC BELIEF PETRI NETS}

Arguments in favor of modeling fuzzy conditional rules by Fuzzy Petri Nets (FPN) can be found in some papers [5], [6]. We assume that the fuzzy knowledge-based logical system is described by fuzzy belief networks with conditional rules and the system modeling is realized by mapping these rules into a Linguistic Belief Petri Net (LBPN). model

Definition 1: A Linguistic Belief Petri Net (LBPN) is a 7-tuple

$$
\mathrm{LBPN}=[\mathrm{P}, \mathrm{T}, \mathrm{I}, \mathrm{O}, \alpha, \Lambda, \mathrm{W}]
$$

where $\mathrm{P}=\left[\mathrm{p}_{1}, \mathrm{p}_{2}, \ldots, \mathrm{p}_{\mathrm{n}}\right]$ denotes a set of places associated with uncertain propositions, $T=\left[\mathrm{t}_{1}, \mathrm{t}_{2}, \ldots, \mathrm{t}_{\mathrm{m}}\right]$ denotes a set of transitions associated with fuzzy implications, $\mathrm{I}(\mathrm{O}): \mathrm{T} \rightarrow \mathrm{P}^{\infty}$ is the input (output) function, a mapping from sets of places to transitions (transitions to set of places). $\alpha: P \rightarrow[0,1]$ is a certainty association function which assigns a linguistic truth value represented as a trapezoidal fuzzy number in $[0,1]$ to each place, $\Lambda: \mathrm{P} \rightarrow[0,1]$ is a certainty threshold association function which assigns a real value $\lambda_{i}$ from zero to one to each place $i$ $\left[\lambda_{1}, \ldots \lambda_{1}, \ldots, \lambda_{\mu}\right]$, For any transition $\mathbf{t}$, if all the trapezoidal fuzzy numbers representing the linguistic certainty factors $\alpha$ associated with the tokens of all its input places have elements greater than their thresholds $\lambda$, then the transition is enabled and fires. $W: T \rightarrow[0,1]$, are a set of weights that assigns a linguistic truth value represented as a trapezoidal fuzzy number in $[0,1]$ to the transition arcs of a net. For a transition $\mathbf{t}, w_{j}$ $\in W$ indicates the linguistic belief value of the implication (rule) for the jth output place, that is, how much the premises impact the belief values of the jth output place, if the transition fires. To map linguistic conditional rules into LBPN, we map propositions as places; the logical implication functions "THEN" between antecedent and consequent propositions as transitions; the impact of belief values of antecedents over the consequent as arcs. 


\section{INFERENCE WITH LINGUISTIC BELIEF PETRI NETS}

A LBPN is an inference engine. The inference engine is activated by firing transitions. A transition $\mathbf{t}$ fires instantly as soon as it is enabled. $\mathbf{t}$ is enabled if all its input places have tokens whose certainty factors are greater than their thresholds.

Let $I(t)=\left[p_{i 1}, p_{i 2}, \ldots, p_{\text {in }}\right]$, with the corresponding thresholds: $\lambda_{1}, \lambda_{2}, \ldots, \lambda_{v}$ and let $\mathrm{O}(\mathrm{t})=\left[\mathrm{p}_{\mathrm{ol}}, \mathrm{p}_{\mathrm{o} 2}, \ldots, \mathrm{p}_{\mathrm{om}}\right]$ with the corresponding output weights: $\mathrm{w}_{\mathrm{O} 1}, \mathrm{w}_{\mathrm{O} 2}, \ldots, \mathrm{w}_{\mathrm{Om}}$,

Definition 2: $\forall \mathbf{t} \in \mathrm{T}, \boldsymbol{t}$ is enabled and fires if $\forall \mathrm{p}_{\mathrm{ij}} \in \mathrm{I}(\mathrm{t})$, the linguistic (trapezoidal fuzzy number) certainty value $\alpha\left(\mathrm{p}_{\mathrm{ij}}\right)$ has an interval $\left[\alpha_{\mathrm{kij}}\left(\mathrm{p}_{\mathrm{ij}}\right)\right]$ such that all its values $\alpha_{\mathrm{kij}} \geq \lambda_{\mathrm{j}}, \mathrm{j}=1,2, \ldots, \mathrm{n}$ After firing $\mathbf{t}$, the token in $\mathrm{p}_{\mathrm{ij}}$ is removed, and a token with a membership certainty function $W_{o k}\left[\min _{j}\left\{\alpha_{k i j}\left(p_{i j}\right)\right\}\right]$ is put into each of its output places $p_{o k}, k=1,2, \ldots, m$. If a place $p_{o k}$ has more than one input transition and more than one of its input transitions fire, then the new certainty factor of $p_{o k}$ is the new token which is produced by the transition with the maximum output weight, because it has the maximal evidence.

In order to describe the fuzzy reasoning algorithm more clearly, we make some other remarks.

Let $\mathrm{P}$ the set of places. Let divide $\mathrm{P}$ into three parts: $\mathrm{P}_{1}, \mathrm{P}_{\text {int }}$ and $\mathrm{P}_{\mathrm{O}}$ such that $\mathrm{P}=\mathrm{P}_{\mathrm{I}} \cup \mathrm{P}_{\text {int }} \cup \mathrm{P}_{\mathrm{O}}$ and $\mathrm{P}_{\mathrm{I}} \cap \mathrm{P}_{\text {int }} \cap \mathrm{P}_{\mathrm{O}}=\varnothing$. Furthermore $P_{I}=\{p \in P \mid \bullet p=\varnothing\}, P_{\text {int }}=\{p \in P \mid \bullet p \neq \varnothing$ and $p \bullet \neq \varnothing\}, P_{O}$ $=\{p \in P \mid p \bullet=\varnothing\}$. Then $p \in P_{1}$ is called an initial premise, $p \in$ $P_{i n t}$ is called a hidden proposition, $p \in P_{O}$ is called a conclusion.

\section{FUZZY REASONING ALGORITHM}

Step 1. Put a token on initial premises $P_{t}$. and define initial transitions $T_{t}$

Step 2. Find the enabled transitions $T_{e}$ from $T_{t}$. That is where $\forall p_{i j} \in P_{I}$ the linguistic (trapezoidal fuzzy number) certainty value $\alpha\left(p_{i j}\right)$ has an interval $\left[\alpha_{k i j}\left(p_{i j}\right)\right]$ such that all its values $\alpha_{k i j} \geq \lambda_{j}, j=1,2, \ldots, n$

Step 3. Fire all enabled transitions $T_{e}$ and calculate new certainty membership functions which are produced by fired transitions according to Definition 2.

Step 4. Make token transmission. Take a token from the input places of a fired transition. Assume $p$ is one of the output places of a fired transition 
4.1 If $p$ has only one input transition, then add a token to $p$ with the certainty membership function $\mathrm{w}_{\mathrm{ok}}\left[\min _{\mathrm{j}}\left\{\alpha_{\mathrm{kij}}\left(\mathrm{p}_{\mathrm{ij}}\right)\right\}\right]$ which is produced by its input transition;

4.2 If If $p$ has more than one input transition and more than one of its input transitions fire, then the new certainty membership function of $p$ is the new token which is produced by the transition with the maximum output weight, because it has the maximal evidence.

Step 5. Let $T=T-T_{e}$, and define the next transitions $T_{t}$ and the places with tokens $P_{t}$.

Step 6. Go to Step 2 and repeat, until $T_{\mathrm{e}}=\varnothing$

\section{EXAMPLE}

Let following knowledge base:

$\mathbf{A}=$ "There is fever"

$\mathbf{B}=$ "There is throat pain"

$\mathrm{C}=$ "The leukocyte count is too large"

$\mathbf{D}=$ "There is a throat infection"

$$
\mathbf{E}=\text { "The patient must stay at home" }
$$

Implication $\mathbf{t}_{1}$ : = "If the patient has fever (more than $38^{\circ} \mathrm{C}$ ) and there is throat pain, then the patient should stay at home with "high" certainty $\left(\mu_{1}=\right.$ "high").

Implication $\mathbf{t}_{2}$ : = "If the patient has fever (more than $38^{\circ} \mathrm{C}$ ) and there is throat pain and the leukocyte count is too large, then the certainty of a throat infection is very high ( $\mu_{2}=$ "very high").

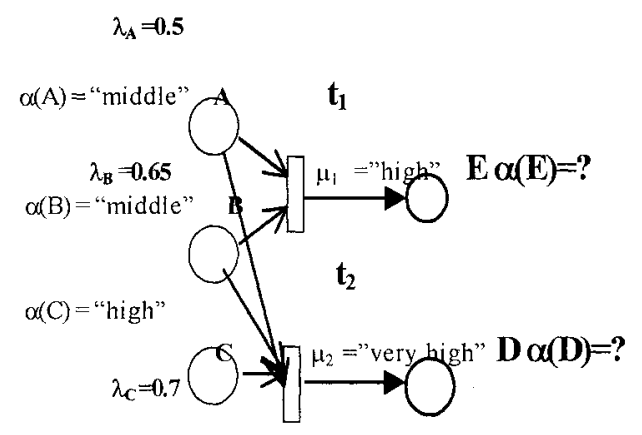

Figure 1 Linguistic Belief Petri Net corresponding to Example 
Let the following facts:

The certainty $\alpha(\mathrm{A})$ of $\mathbf{A}$ is "middle". Its threshold is 0.5

The certainty $\alpha(B)$ of $\mathbf{B}$ is "middle". Its threshold is 0.65

The certainty $\alpha(C)$ of $\mathbf{C}$ is "high". Its threshold is 0.7

It is asked the certainty value $\alpha(E)$ of $\mathbf{E}=$ "The patient must stay at home" and $\alpha(D)$ of $\mathbf{D}=$ "There is a throat infection".

Let following linguistic terms and their corresponding fuzzy numbers.

\begin{tabular}{|l|c|}
\hline Linguistic terms & $\begin{array}{c}\text { Trapezoidal Fuzzy } \\
\text { Numbers }\end{array}$ \\
\hline Absolutely false & $(0,0,0,0)$ \\
\hline Low certainty & $(0,1,2,3)$ \\
\hline Middle certainty & $(2,4,6,8)$ \\
\hline High certainty & $(7,8,9,1)$ \\
\hline Very High certainty & $(9,1,1,1)$ \\
\hline
\end{tabular}

This table can be represented in Fig 2

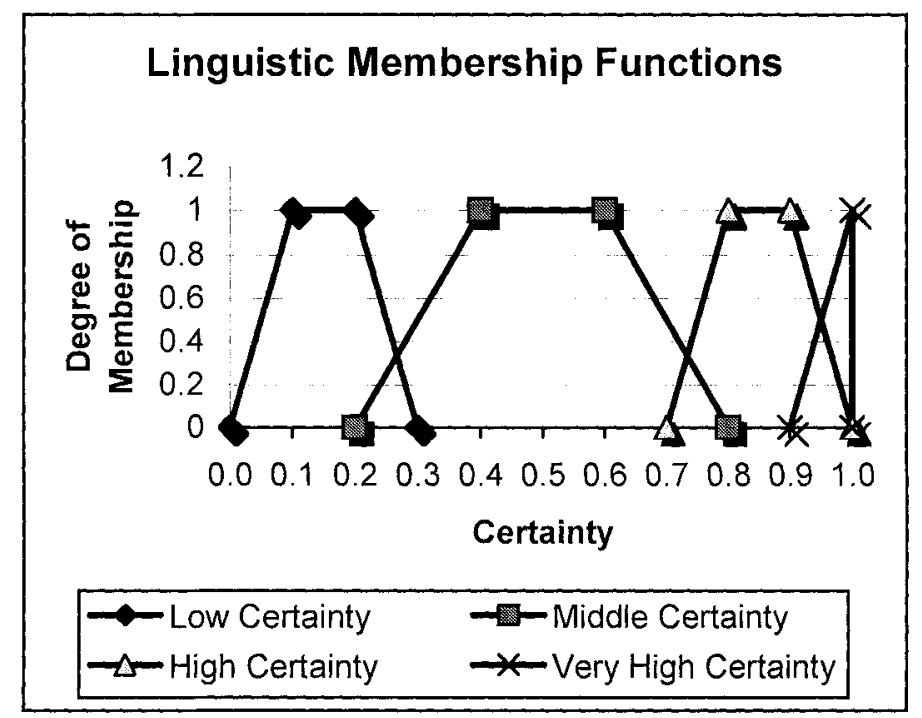

Figure 2. Trapezoidal Membership Functions of Linguistic Certainty Values 
Step 1. Initial premises are $A, B$ and $C . P_{t}=A \cup B \cup C . T_{t}=\left[t_{1}, t_{2}\right]$

Step 2. $\alpha(A)=$ "middle" and its fuzzy membership has values greater than its thresholod 0.5 In the same way $\alpha(B)=$ "middle" and its fuzzy membership has values greater than its threshold 0.6 then transition $t_{1}$ is enabled. Also $\alpha(C)=$ "high" and its fuzzy membership has values greater than its threshold 0.7 then transition $\mathrm{t}_{2}$ is enabled too.

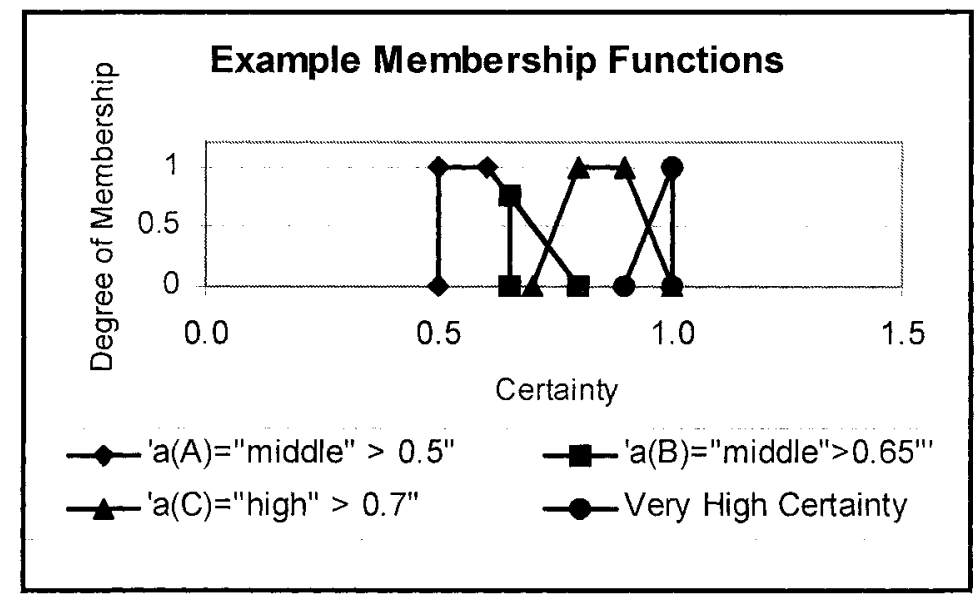

Figure 3. Trapezoidal Membership Functions of Example Certainty Values

Step 3. Transition $t_{1}$ fires. After firing $t_{1}$ a membership certainty function "high" $\otimes[\min \{("$ middle" $>0.5)$, ("middle" $>0.65)\}]$ is calculated for $\mathrm{E}$. The way to calculate this is membership function is to consider that "high" is represented by $(0.7,0.8,0.9,1)$, "middle" $>0.5$ is represented by $(0.5,0.5$, $0.6,0.8)$, "middle" $>0.65$ is represented by $(0.65,0.65,0.65,0.8)$. Therefore, the certainty membership function associated with $\mathrm{t}_{1}$ and $\mathrm{E}$ is $(0.7,0.8,0.9,1)$ $\otimes[\min \{(0.5,0.5,0.6,0.8),(0.65,0.65,0.65,0.8)\}]=(0.7,0.8,0.9,1) \otimes$ $(0.65,0.65,0.65,0.8)=(0.455,0.520,0.585,0.8)$.

In the same way, transition $t_{2}$ fires. After firing $t_{2}$ a membership certainty function "very high" $\otimes[$ min $\{("$ middle" $>0.5)$, ("middle" $>0.65)$, ("high" $>0.7)\}]$ is calculated for D.

Its value is given by $(0.9,0.9,1,1) \otimes[\min \{(0.5,0.5,0.6,0.8),(0.65$, $0.65,0.65,0.8),(0.7,0.8,0.9,1)\}]=(0.9,0.9,1,1) \otimes[(0.5,0.5,0.6,0.8)]$ $=(0.45,0.45,0.6,0.8)$. 
Step 4. A token is taken from inputs A, B and C. E receives one token with trapezoidal certainty function $(0.455,0.520,0.585,0.8)$. D receives one token with trapezoidal certainty function $(0.45,0.45,0.6,0.8)$.

Step 5. Let $T=T-T_{e}=\varnothing$ There is no more transitions $T_{t}$. The places with tokens $P_{t}$ are $E$ and $D$.

Step 6. Go to Step2. There are no more enabled transitions $T_{e}=\varnothing$. The conclusions are: $\mathbf{E}=$ "The patient must stay at home" with trapezoidal certainty function $(0.455,0.520,0.585,0.8)$ that could be translated into linguistic terms as "Probably the patient must stay at home" and $\mathrm{D}=$ "There is a throat infection" with trapezoidal certainty function $(0.45,0.45$, $0.6,0.8)$ that could be translated into linguistic terms as "It could be that the patient has a throat infection". Their graphics are represented in Figure 4.

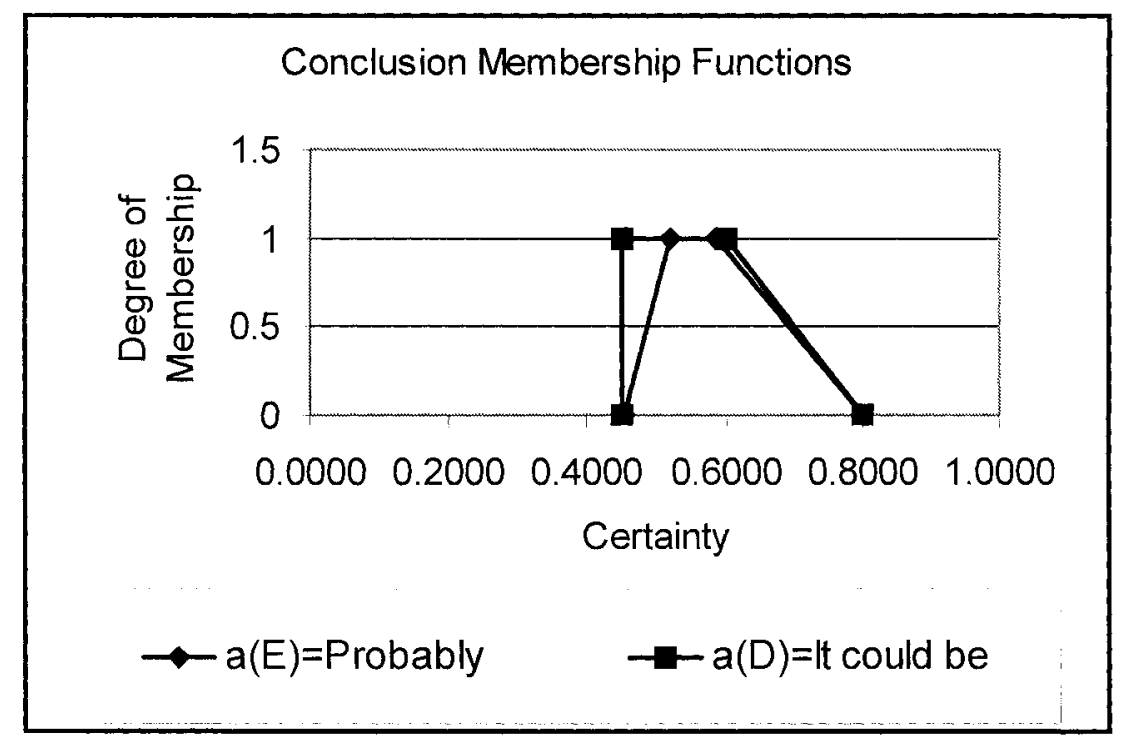

Figure 4 Certainty Functions of the Conclusions

\section{CONCLUSION}

Expert Consultation Techniques are based not on trying to model the real world, but to extract the conceptual model that an expert or a panel of experts have about it, that permits them with remarkable efficiency to solve real world problems in their speciality domains. Aiming at this objective, a 
fuzzy logical knowledge based model was proposed, implemented as a generalized fuzzy Petri net model, to make inferences and to solve problems.

\section{REFERENCES.}

[1] Linstone, H. A. \& Turoff, M. (1972). "The Delphi Method". New York: Universe Books.

[2] Yager R R, (1992) "Expert Systems using Fuzzy Logic" in Yager R R \& Zadeh L A (eds) "An Introduction to Fuzzy Logic Applications in Intelligent Systems". Boston: Kluwer Academic Publishers.

[3] Lara-Rosano F. (1989) "Uncertain Knowledge Representation through Fuzzy Knowledge Networks based on Lukasiewicz Logic", in Advances in Computer Science, George E. Lasker (ed), Windsor, Canada: IIAS, ISBN 0921836-031: 32-38

[4] Zadeh L A (1965) "Fuzzy Sets", Information and Control, 8: 338-353

[5] Yeung D.S. and Tsang E.C.C. (1994) "Fuzzy knowledge representation and reasoning using Petri nets", Expert Systems \& Applications, 7: 281-290

[6] Xiaoou Li \& Lara-Rosano F. (2000) "Adaptive Fuzzy Petri Nets for Dynamic Knowledge Representation and Inference", "Expert Systems with Applications", 19(3): 235-241 . 